\title{
Automobile et environnement : contribution de la recherche aérodynamique à la réduction des gaz à effet de serre
}

\author{
PATRick Gilliéron ${ }^{1,2}$ ET Azeddine Kourta ${ }^{1,2, a}$ \\ 1 Renault, Direction de Recherche (DREAM), 1 avenue du Golf, 78288 Guyancourt Cedex, France \\ 2 ESA/Institut PRISME, Polytech'Orléans 8, rue Léonard de Vinci, 45072 Orléans Cedex, France
}

Reçu le 30 avril 2008, accepté le 20 décembre 2008

\begin{abstract}
Résumé - Cet article traite du contrôle des décollements aérodynamiques pour réduire la consommation et les émissions de gaz à effet de serre des futurs véhicules automobiles. Après avoir passé en revue les moyens de contrôle passif, des exemples de contrôle actif par aspiration, soufflage ou par jet synthétique sont successivement présentés. Les résultats montrent le fort potentiel des solutions présentées. Des réductions de traînée proches de $20 \%$ sont ainsi déjà envisagées sans que soit nécessaire de contraindre les géométries actuelles en termes d'habitabilité et de confort. Les coefficients de traînée aérodynamique des futurs véhicules automobiles destinés aux liaisons extra urbaines pourraient alors très prochainement tendre vers des valeurs voisines de 0,24. Cette évolution permettrait alors de réduire de 1,6 l sur $100 \mathrm{~km}$ la consommation moyenne d'un véhicule de moyenne gamme se déplaçant sur autoroute à la vitesse de $130 \mathrm{~km} . \mathrm{h}^{-1}$. La réduction des émissions de gaz carbonique serait au moins de l'ordre de $7 \mathrm{~g} . \mathrm{km}^{-1}$ suivant le cycle NEDC (New European Driving Cycle).
\end{abstract}

Mots clés : Traînée / contrôle / gaz effet de serre / automobile

\begin{abstract}
Automotive and environment: aerodynamic research contribution to the greenhouse gas reduction. This paper deals with the aerodynamic separation control to reduce consumption and the greenhouse gas emission for the future road vehicles. After reviewing the passive control methods, examples of active flow control by using suction, blowing and synthetic jets are successively presented. Drag reductions of about $20 \%$ are hence already possible without any negative geometrical modification in term of habitability and comfort. Drag reduction coefficients of the future road vehicles doing outside city connections could soon tend to a value near 0.24 . The result must allow to reduce the fuel consumption of a vehicle of the range M1 (vehicle of the lower average range) of approximately 1.6 liters by $100 \mathrm{~km}$ at the speed of $130 \mathrm{~km} \cdot \mathrm{h}^{-1}$. The $\mathrm{CO}_{2}$ emission reduction will be at least about $7 \mathrm{gr} . \mathrm{km}^{-1}$ following NEDC (New European Driving Cycle).
\end{abstract}

Key words: Drag / control / greenhouse gases / automotive

\section{Introduction}

Le parc automobile mondial comptait en 2002 près de 590 millions d'unités dont 182 en Europe de l'Ouest, 140 aux États-Unis, 55 au Japon, 29 en France, 9 en Chine et 6 en Inde [1]. Dans ce contexte, les émissions annuelles de $\mathrm{CO}_{2}$ dépassent déjà les 22 milliards de tonnes alors que les besoins en transports des pays émergents augmentent. À titre d'exemple, la demande en énergie fossile de la Chine et de l'Inde transforme aujourd'hui le système énergétique mondial qui devient incompatible avec les ressources disponibles et la nécessité de réduire les émissions de gaz à effet de serre. Le parc automobile

a Auteur pour correspondance :

azeddine.kourta@univ-orleans.fr chinois pourrait ainsi compter dans 20 ans près de 270 millions de véhicules, soit trente fois plus qu'en 2002. L'Europe de l'Est et l'Afrique suivent le même processus dans des proportions plus ou moins équivalentes et à plus ou moins long terme. Dans ce contexte, les émissions de gaz à effet de serre auront augmenté de près de $57 \%$ en 2030 avec des répercussions fortes sur l'environnement et le climat [2]. Des solutions sont alors recherchées dans différents domaines de la physique, par et pour l'industrie des transports, afin de réduire de manière significative les émissions de dioxyde de carbone. Dans ce contexte et quelle que soit la source d'énergie utilisée (combustible fossile, électrique, hydrogène... ), le contrôle des écoulements aérodynamiques pour les déplacements automobiles interurbains constitue une nécessité pour optimiser l'énergie embarquée. La communauté scientifique 


\section{Nomenclature}

\begin{tabular}{|c|c|}
\hline$d$ & Distance entre deux positions d'aspiration $(\mathrm{m})$ \\
\hline$e$ & Largeur de la fente de soufflage, d'aspiration ou de jet synthétique (m) \\
\hline$l$ & Longueur de la lunette arrière $(\mathrm{m})$ \\
\hline$C_{\mathrm{p}}$ & Coefficient de pression statique \\
\hline$C_{\mathrm{pi}}$ & Coefficient de perte de pression d'arrêt \\
\hline$\omega$ & Vorticité $\left(\mathrm{s}^{-1}\right)$ \\
\hline$C_{\mu}$ & Coefficient de quantité de mouvement \\
\hline$F_{\mathrm{k}}$ & Fréquence caractéristique $(\mathrm{Hz})$ \\
\hline$F_{\mathrm{j}}$ & Fréquence du jet synthétique $(\mathrm{Hz})$ \\
\hline$F^{+}$ & Fréquence réduite $=F_{j} F_{k}^{-1}$ \\
\hline$V_{0}$ & Vitesse de l'écoulement infini amont $\left(\mathrm{m} . \mathrm{s}^{-1}\right)$ \\
\hline$V_{\text {asp }}$ & Vitesse d'aspiration $\left(\mathrm{m} . \mathrm{s}^{-1}\right)$ \\
\hline$V_{\mathrm{s}}$ & Vitesse soufflage $\left(\mathrm{m} . \mathrm{s}^{-1}\right)$ \\
\hline$C_{x}$ & Coefficient de traînée aérodynamique \\
\hline$\Delta C_{x}$ & Variation du coefficient de traînée aérodynamique en $\%$ \\
\hline$\lambda_{2}$ & Critère d'analyse pour la dynamique des tourbillons cohérents \\
\hline \multicolumn{2}{|r|}{$\begin{array}{l}\text { Cycle NEDC pour New European Driving Cycle, cycle d'homologation de tout véhicule léger en Europe. Démonstrateurs } \\
\text { EVE et VERA : premiers prototypes automobiles aérodynamiques développés par Renault et Peugeot au début des années } 80 \\
\text { en réponse au défi proposé par le Ministère de l'Industrie qui souhaitait que soit développée une voiture capable de consommer } \\
\text { moins de } 31 \text { aux } 100 \mathrm{~km} \text {. Le projet EVE a abouti au prototype Vesta II qui le } 23 \text { juin } 1987 \text { a réalisé un record mondial de } \\
\text { consommation sur autoroute entre Bordeaux et Paris. Valeur de Swirl : rapport entre les vitesses azimutale (à la périphérie } \\
\text { d'un tourbillon longitudinal) et axiale (dans la direction de l'axe du tourbillon). } \\
\text { MEMS : pour Micro Electro Mechanical Systems. }\end{array}$} \\
\hline
\end{tabular}

et technique travaille et s'organise alors pour réduire de $30 \%$ la traînée aérodynamique des véhicules et diminuer ainsi de 12 (cycle NEDC pour New European Driving Cycle) à $24 \mathrm{~g}$ (profil client) par kilomètre les émissions de dioxyde de carbone. Cet article propose une présentation des principales voies de progrès pour l'aérodynamique automobile basée sur le maintien des critères actuels d'habitabilité, de confort et de sécurité.

\section{La problématique}

L'objectif de réduction de traînée est nécessaire et ambitieux. Des travaux de recherche sont alors conduits depuis plus de 30 ans pour améliorer la connaissance et contrôler les mécanismes physiques à l'origine de la traînée aérodynamique. Les réductions de traînée aérodynamique associées au rétrécissement des sections d'entrée d'air, à l'accroissement des pertes de charge des échangeurs, à l'extension verticale de la partie basse du bouclier avant ou encore à la présence d'un déflecteur de soubassement positionné en amont du train arrière sont ainsi mieux comprises et utilisées avec plus d'efficacité [3]. Des bilans intégraux de quantité de mouvement permettent d'identifier des voies de progrès pour réduire la contribution du compartiment moteur dans la traînée aérodynamique et améliorer l'efficacité des échangeurs [4-6]. Les écoulements en proches parois sont modélisés à partir de modèles mathématiques pour améliorer la compréhension des portraits de phases relevés lors des visualisations pariétales [7], orienter l'action des ingénieurs et identifier des voies de progrès destinées à réduire la contribution des décollements dans la traînée aérodynamique globale. L'ensemble des progrès accomplis permet aujourd'hui de mieux comprendre les origines de la traînée aérodynamique, d'optimiser les performances aérodynamiques et de mieux intégrer les contraintes Design aux impératifs de réduction de traînée. Ces progrès ne permettent hélas pas de réduire de manière significative la traînée aérodynamique des véhicules automobiles et les coefficients de traînée aérodynamique $C_{x}$, aujourd'hui de l'ordre de 0,30, n'évoluent plus depuis 20 ans.

Particulièrement sensible aux problèmes environnementaux et à l'épuisement des ressources fossiles, la communauté européenne fixe aujourd'hui un objectif clair et ambitieux aux constructeurs automobiles en termes d'émission de gaz à effet de serre. Les rejets de gaz carbonique ne devront ainsi pas dépasser 120 g. $\mathrm{km}^{-1}$ à l'horizon 2012 et sans doute $90 \mathrm{~g}$ vers 2018. Des solutions en rupture doivent alors être imaginées pour réduire d'au moins $20 \%$ la traînée aérodynamique d'ici l'échéance 2012, c'est-à-dire d'ici quatre ans. Pour l'utilisateur final et $100 \mathrm{~km}$ parcourus à $130 \mathrm{~km} \cdot \mathrm{h}^{-1}$, l'objectif consiste à réduire la consommation de carburant d'un véhicule de la gamme M1 (véhicule de la gamme moyenne inférieure) d'environ 1,6 l. Sur le parcours mixte normalisé dit NEDC (pour New European Driving Cycle), la réduction des émissions de $\mathrm{CO}_{2}$ serait de l'ordre de $0,7 \mathrm{~kg}$ pour $100 \mathrm{~km}$ parcourus soit $7 \mathrm{~g} \cdot \mathrm{km}^{-1}$. 


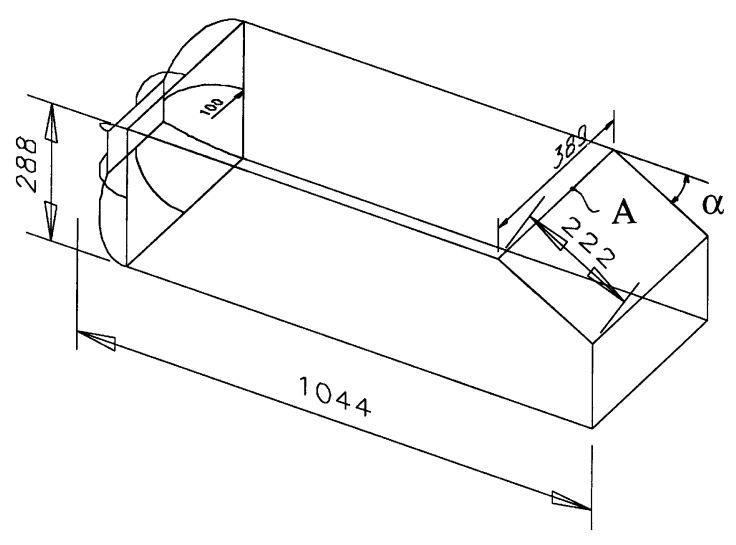

Fig. 1. Géométrie du corps de Ahmed, échelle 1. Notations : A, fin de pavillon; $\alpha$ angle de lunette arrière/horizontale [8].

\section{Les solutions de rupture}

Un véhicule automobile est un corps de faible allongement qui se déplace à proximité du sol dans un écoulement naturellement turbulent. L'écoulement décolle au niveau des entrées d'air, au niveau du soubassement, autour de la roue, dans les passages de roues, au niveau du pied de pare-brise, des montants latéraux avant et enfin au niveau de la lunette arrière et du culot. La contribution des contraintes de frottement reste faible, de l'ordre de $10 \%$, et la traînée aérodynamique résulte principalement de la distribution des contraintes de pression réparties sur la lunette arrière et le culot. Ces contraintes sont classiquement à l'origine de $90 \%$ de la traînée aérodynamique globale et sont à $80 \%$ concentrées sur les parties arrière. Les interactions entre les différentes régions du véhicule sont nombreuses, complexes et la recherche de solutions efficaces de contrôle nécessite de travailler sur des géométries simplifiées capables de reproduire l'essentiel des décollements et phénomènes physiques rencontrés sur un véhicule réel. La géométrie proposée par Ahmed en 1984 a donc été retenue depuis plus de 10 ans par l'ensemble de la communauté scientifique pour analyser les phénomènes, rechercher et mettre au point de nouvelles solutions de contrôle destinées à réduire la traînée aérodynamique [8]. Cette géométrie simplifiée de type bicorps (pas de coffre arrière) permet de reproduire l'essentiel des décollements qui apparaissent sur une géométrie réelle de véhicule automobile, voir figure 1.

Sur cette géométrie, l'écoulement décolle en fin de pavillon pour donner naissance à une nappe décollée ouverte qui recolle en moyenne vers le bas de la lunette arrière. L'écoulement décollé sous la nappe est le siège de deux mouvements contrarotatifs qui convergent vers deux foyers de séparation, origine du cornet B sur la figure 1 et son symétrique par rapport au plan $y=0[9,10]$. L'écoulement issu de ces deux foyers interagit et alimente ensuite l'écoulement de culot. Une partie de l'écoulement issu des parties latérales de la géométrie décolle au niveau des arêtes latérales de lunette arrière et donne naissance à deux structures tourbillonnaires longitudinales contrarotatives. L'autre partie alimente l'écoulement situé sous

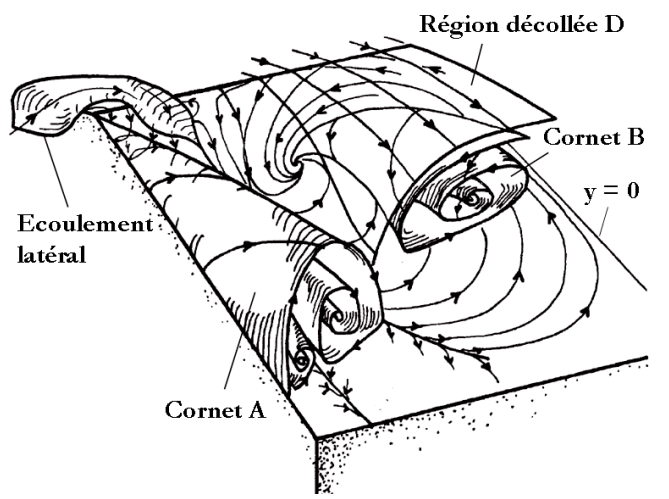

Fig. 2. Représentation topologique schématique de la structure décollée de la partie gauche de la lunette arrière, vu de l'arrière, d'après [10].

la région décollée de fin de pavillon, évolue vers le bas de la lunette arrière, le plan de symétrie $y=0$ puis remonte vers le haut en spiralant vers l'origine du cornet $\mathrm{B}$, figure 2. Au culot, l'écoulement décolle à la périphérie de la géométrie et donne naissance à une structure tourbillonnaire en forme de tore qui interagit avec l'écoulement décollé de lunette arrière. Les résultats expérimentaux montrent que la topologie de l'écoulement reste identique et que la traînée aérodynamique augmente continûment avec l'accroissement de l'angle d'inclinaison de la lunette arrière entre 12 et $30^{\circ}$.

\subsection{Résultats en contrôle passif}

Le contrôle passif des écoulements et des décollements s'effectue à partir de solutions qui ne nécessitent aucune puissance à prendre sur le générateur principal d'énergie du véhicule. Ce contrôle s'effectue ainsi de manière classique à partir des connaissances mises en évidence et capitalisées entre 1976, date de première mise en service de la soufflerie S4 (IAT Saint Cyr) pour automobile, et 1985. Durant cette période, les coefficients moyens de traînée aérodynamique des véhicules automobiles ont été réduits d'un tiers, (de 0,45 à 0,30 ) et des réductions plus importantes obtenues sur les démonstrateurs EVE (Renault) et VERA (Peugeot), voir nomenclature. Les solutions identifiées sont toujours utilisées mais restent très contraintes par les exigences du marché en termes de confort, d'habitabilité et de sécurité. Les meilleurs coefficients de traînée aérodynamique demeurent ainsi proches de 0,30 depuis plus de 20 ans. Pour améliorer ce résultat, des solutions nouvelles basées sur des géométries en rupture et une meilleure connaissance des processus de formation et de développement de la traînée aérodynamique doivent être recherchées.

Des dispositifs de contrôle ont alors été développés ces dernières années pour élargir l'offre des solutions aérodynamiques proposées aux ingénieurs et designers automobiles. Des réductions de traînée significatives ont ainsi été obtenues à partir d'éléments transversaux positionnés en aval et en amont d'une géométrie de forme 


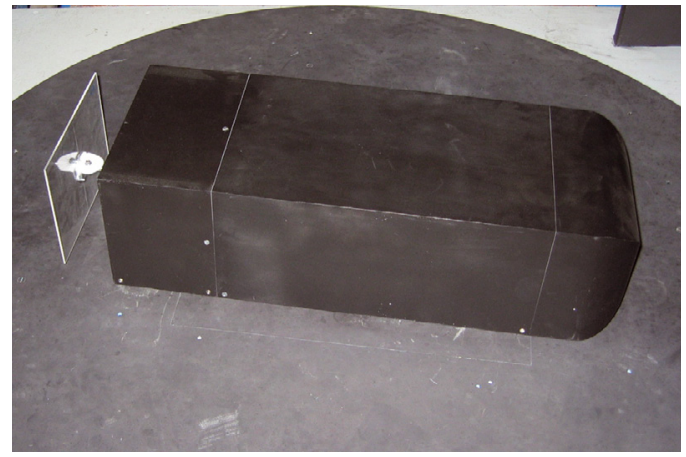

Fig. 3. Élément séparateur transversal sur la géométrie simplifiée désignée corps de Ahmed [11].

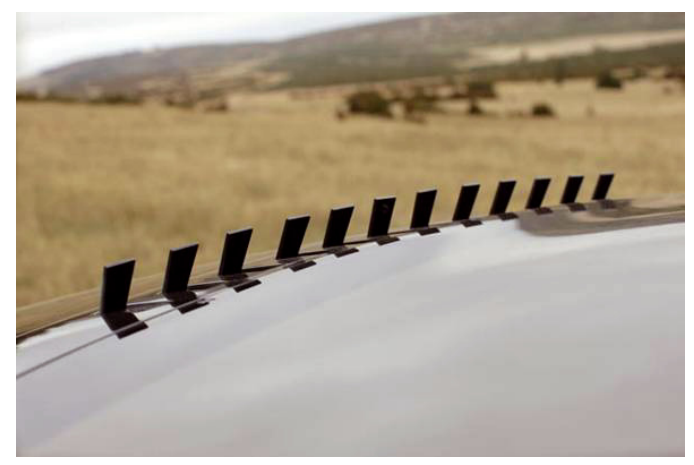

Fig. 4. Générateurs de séparation sur concept car Airlounge PSA (avec l'aimable autorisation de PSA).

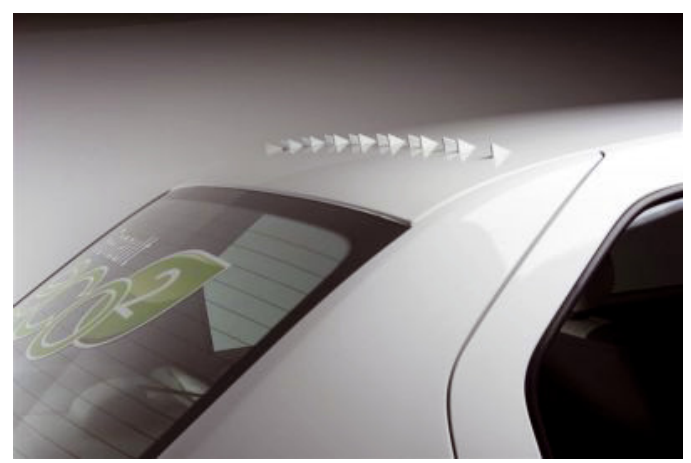

Fig. 5. Générateurs de vortex sur véhicule Logan Dacia/Renault [12].

anguleuse [11] ainsi qu'à partir de générateurs de vortex mécaniques [12], figures 3-5. Des travaux sont également engagés pour tester la capacité des milieux poreux à réduire la traînée aérodynamique et des réductions proches de $45 \%$ ont été obtenues sur une géométrie simplifiée bidimensionnelle à culot droit [13]. Ces solutions offrent des alternatives intéressantes d'un point de vue aérodynamique qui restent industriellement inexploitées à ce jour.

\subsection{Résultats en contrôle actif}

Le contrôle actif des écoulements et des décollements s'effectue à l'aide d'actionneurs dont le fonctionnement

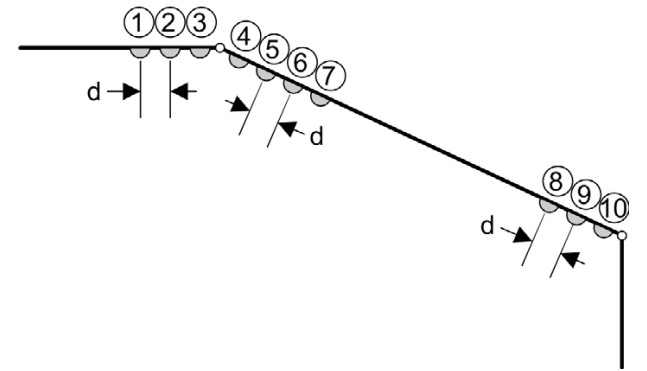

Fig. 6. Positions des fentes d'aspiration en fin de pavillon, sur le haut et en fin de la lunette arrière du corps de Ahmed. La largeur des fentes est identique quelle que soit la configuration et la distance $d$ est égale à $10^{-2} \mathrm{~m}[16]$.

nécessite une puissance à prendre généralement sur le générateur principal d'énergie du véhicule. La partie visible de ces systèmes est constituée d'orifices circulaires ou de fentes distribuées sur la surface du véhicule au voisinage de l'écoulement à contrôler. Leur fonctionnement s'effectue à partir de systèmes mécaniques, électromagnétiques ou électriques logés dans les parties creuses de la structure du véhicule. Leurs masses et leurs encombrements doivent être les plus faibles possibles pour réduire leurs impacts sur la consommation et les volumes utiles ou habitables.

Plusieurs solutions de contrôle ont été identifiées, testées et analysées par différents auteurs pour le contrôle des écoulements et des décollements en aéronautique, hydrodynamique et aérodynamique des véhicules terrestres. Les solutions retenues consistent généralement en des systèmes qui permettent de souffler ou aspirer de manière continue ou alternée au travers d'orifices circulaires ou rectangulaires.

\subsubsection{Solutions par aspiration continue}

Le contrôle des décollements et de la traînée aérodynamique des véhicules terrestres peut s'effectuer à partir d'aspiration continue au travers de fentes transversales disposées en amont ou en aval de la ligne de décollement. Les expérimentations numériques effectuées sur un corps de Ahmed muni d'une lunette arrière inclinée à $25^{\circ}$ à partir d'une fente disposée en envergure et de largeur $e$ permettent de mettre en évidence les résultats suivants [14].

(i) Position de la fente par rapport à la position $d u$ décollement : pour des expérimentations effectuées en $2 \mathrm{D}$, les réductions maximales de traînée aérodynamique s'obtiennent pour des fentes positionnées au voisinage immédiat et de part et d'autre de la ligne de décollement. Les réductions de traînée sont quantitativement identiques mais moins sensibles à la position longitudinale lorsque la fente est positionnée en aval du décollement, figures 6 et 7 .

(ii) Influence de la vitesse d'aspiration : l'efficacité du contrôle sur la réduction de la traînée aérodynamique 


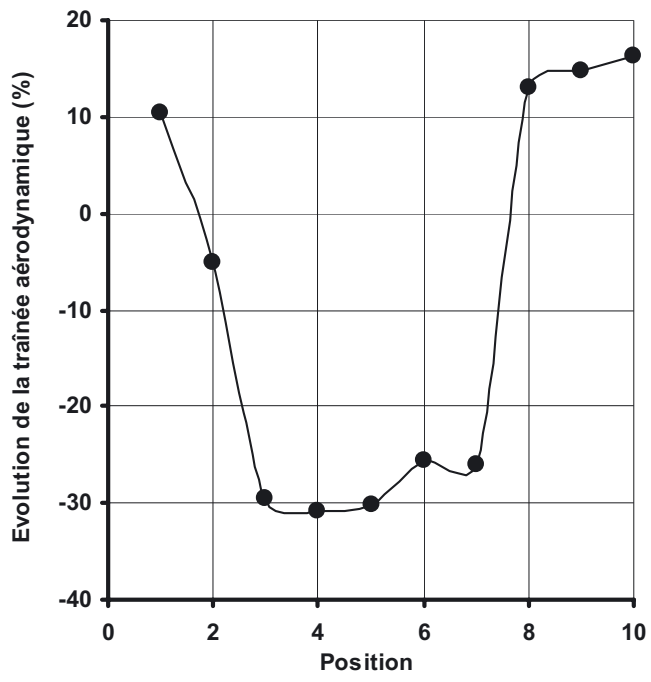

Fig. 7. Influence de la position de la fente sur la réduction du coefficient aérodynamique $C_{x}$, résultats numériques $2 \mathrm{D}$ corps de Ahmed [16].

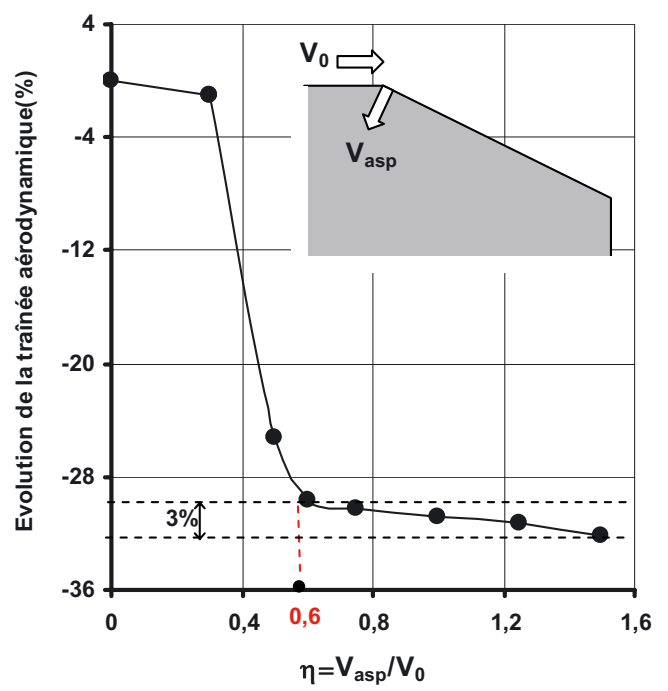

Fig. 8. Influence de la vitesse d'aspiration sur la réduction du coefficient de traînée aérodynamique $C_{x}$, résultats numériques 2D corps de Ahmed [16].

augmente rapidement avec l'accroissement de la vitesse d'aspiration. La traînée aérodynamique diminue ainsi de $30 \%$ lorsque le rapport de la vitesse d'aspiration $V_{\text {asp }}$ sur la vitesse infinie amont $V_{0}$ devient égal à 0,6 . Pour des rapports supérieurs, le gain de traînée continue faiblement d'augmenter mais l'efficacité énergétique cesse d'être intéressante, figure 8.

(iii) Influence de l'inclinaison de la direction d'aspiration : l'influence de l'orientation de la vitesse d'aspiration par rapport au plan de lunette arrière est analysée pour des angles compris entre 10 et $145^{\circ}$ et une vitesse d'aspiration égale à $0,6 V_{0}$. Pour les angles testés, la meilleure réduction de traîne, de l'ordre de $30 \%$, s'obtient pour une aspiration normale, figure 9 .

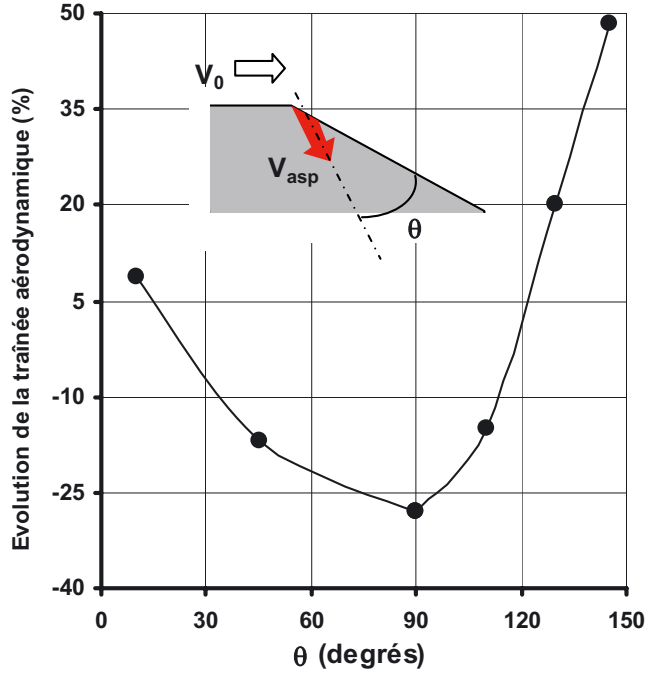

Fig. 9. Influence de l'orientation de la vitesse d'aspiration sur la réduction du coefficient de traînée aérodynamique $C_{x}$, résultats numériques $2 \mathrm{D}$ corps de Ahmed [16].

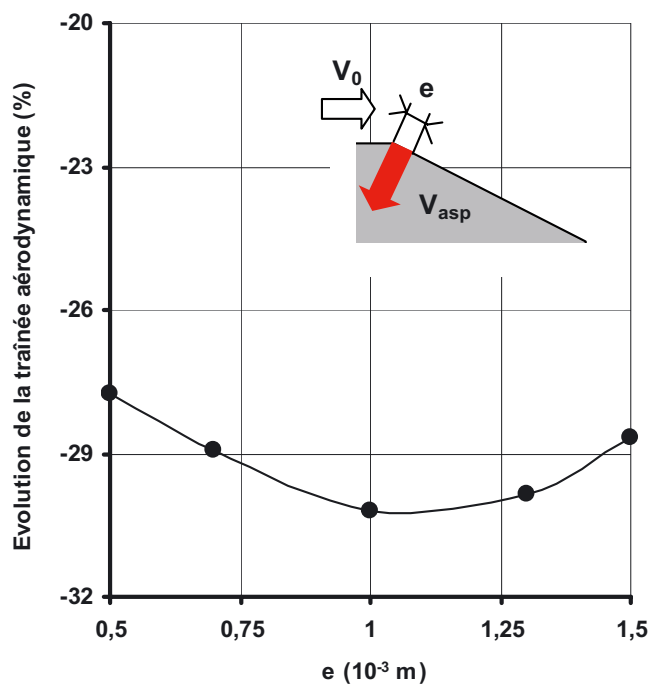

Fig. 10. Influence de la largeur de la fente d'aspiration sur la réduction du coefficient de traînée aérodynamique $C_{x}$, résultats numériques $2 \mathrm{D}$ corps de Ahmed [16].

(iv) Influence de la largeur de la fente : l'influence de la largeur de la fente est analysée pour une fente d'aspiration positionnée immédiatement en aval du décollement, une vitesse d'aspiration égale à 0,6 fois la vitesse de l'écoulement infinie amont et une aspiration dirigée suivant la normale à la lunette arrière. Pour des largeurs de fente comprises entre 0,5 et $1,5 \times 10^{-3} \mathrm{~m}$, la meilleure réduction de traînée est relevée pour une largeur de fente égale à $10^{-3} \mathrm{~m}$, figure 10 .

Appliqués sur une géométrie 3D du corps de Ahmed, les meilleurs paramètres relevés lors de l'étude bidimensionnelle permettent d'obtenir des réductions de traînée proches de 18 \% [15]. Pour cette configuration, le décollement de fin de pavillon disparaît et la distribution 


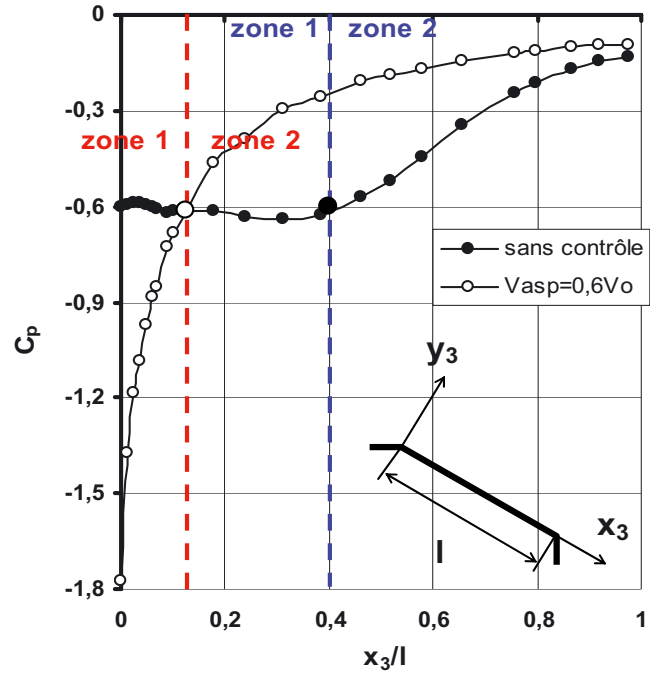

Fig. 11. Influence du contrôle normal en position 4 (Fig. 6) sur les coefficients de pression statique $C_{\mathrm{p}}$ de lunette arrière. Résultats numériques 3D corps de Ahmed, plan $y=0$ [16].

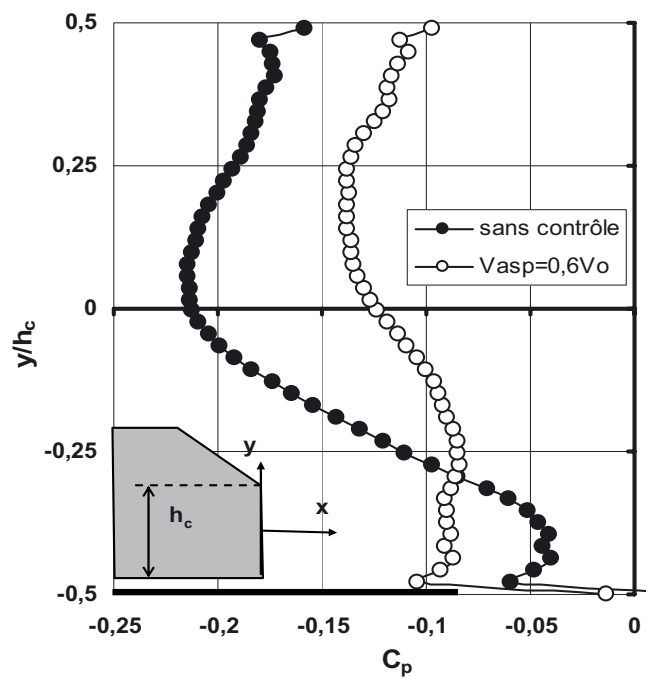

Fig. 12. Influence du contrôle normal en position 4 (Fig. 6) sur la distribution des coefficients de pression statique $C_{\mathrm{p}}$ au culot. Résultats numériques 3D corps de Ahmed, plan $y=0$ [16].

des coefficients de pression statique sur la lunette et au culot évolue de manière significative, figures 11 et 12 .

Sur la lunette arrière, les coefficients de pression statique diminuent très fortement puis augmentent progressivement jusqu'à atteindre des valeurs supérieures aux valeurs relevées sans contrôle au bas de la lunette arrière, figure 11. Au culot, le contrôle par aspiration tend à uniformiser la distribution des pressions statiques et accroître sa valeur moyenne, figure 12. Des expérimentations effectuées en soufflerie sur une géométrie identique munie d'une fente d'aspiration positionnée sur le haut de la lunette arrière confirment ces résultats [16].

L'aspiration peut aussi être utilisée pour réduire la contribution des structures tourbillonnaires de montant de baie dans la traînée aérodynamique. L'évolution des

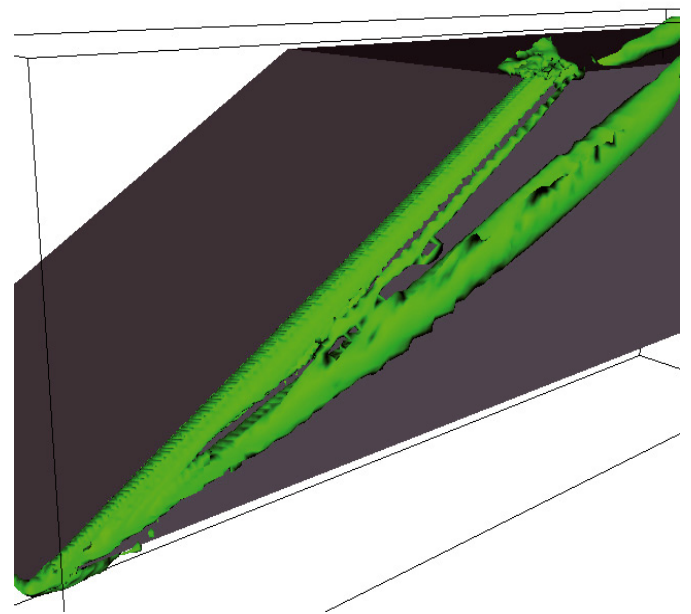

Fig. 13. Pare-brise simplifié sans aspiration, visualisation isosurface $\lambda_{2}=-25 \mathrm{~s}^{-2}$. Vue de $3 / 4$ avant droite [18]. Résultats numériques $3 \mathrm{D}$ montants de baie simplifiés.

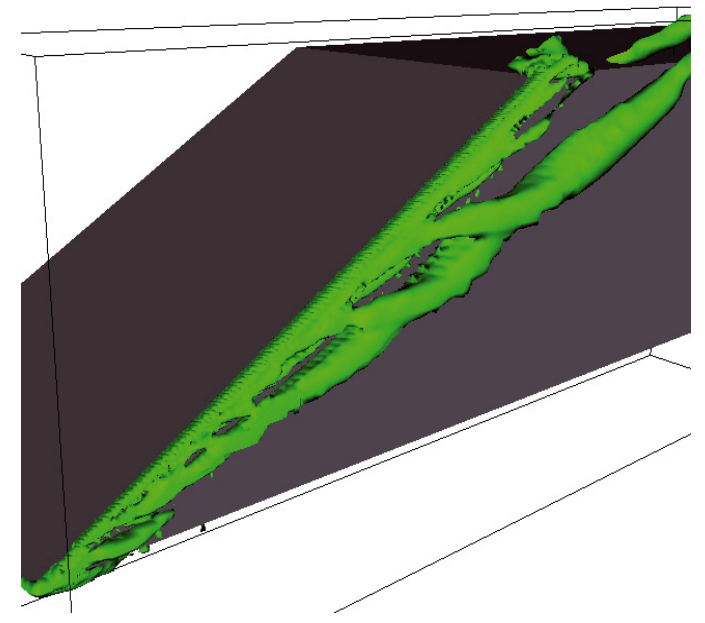

Fig. 14. Pare-brise simplifié avec aspiration $V_{\text {asp }}=-0,5 V_{0}$, visualisation iso-surfaces $\lambda_{2}=-25 \mathrm{~s}^{-2}$. Vue de $3 / 4$ avant droite [18]. Résultats numériques 3D montants de baie simplifiés.

structures tourbillonnaires de montants de baies obtenues par calculs et relevées sans et avec aspiration pour les vitesses $0,5 V_{0}$ et $V_{0}$ est reportée sur les figures 13 à 15. Pour la configuration géométrique retenue et des structures tourbillonnaires représentées à partir du critère $\lambda_{2}$, l'influence de l'aspiration n'est significative qu'au-delà de la vitesse d'aspiration $V_{\text {asp }}=0,5 V_{0}$. Des réductions de traînée proches de $14 \%$ sont ainsi relevées par voies numérique et expérimentale $[17,18]$. Ce résultat s'obtient pour une direction d'aspiration normale à la paroi latérale positionnée immédiatement en aval de la ligne de décollement, une vitesse d'aspiration égale à la vitesse de l'écoulement infini amont et une fente de largeur $e=10^{-3} \mathrm{~m}$ positionnée sur toute la hauteur du montant de baie. L'analyse des résultats montre des modifications importantes au niveau des distributions 


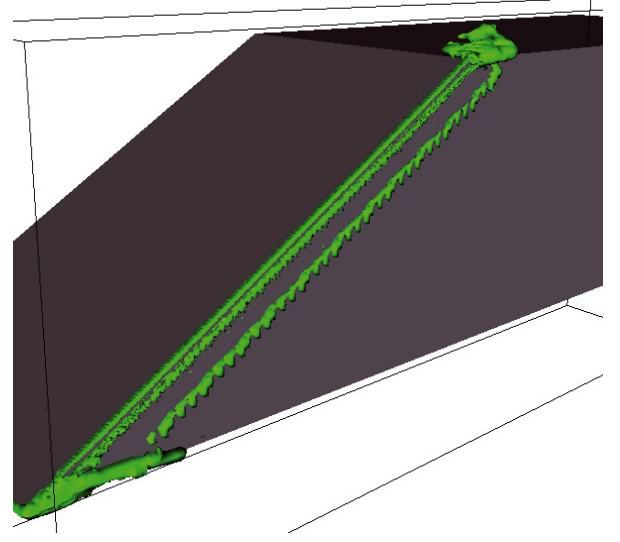

Fig. 15. Pare-brise simplifié avec aspiration $V_{\text {asp }}=-V_{0}$, visualisation iso-surfaces $\lambda_{2}=-25 \mathrm{~s}^{-2}$. Vue de $3 / 4$ avant droite [18]. Résultats numériques 3D montants de baie simplifiés.

surfaciques des pressions pariétales instantanées mesurées sur les surfaces latérales (vitres latérales des véhicules automobiles) [19]. Ce dernier résultat constitue à lui seul une perspective intéressante pour diminuer le bruit d'origine aéroacoustique, améliorer la stabilité dynamique du véhicule lors des phases transitoires ou réduire les salissures.

\subsubsection{Solution par soufflage continu}

Le contrôle des décollements et de la traînée aérodynamique des véhicules terrestres peut aussi s'effectuer par soufflage continu. Dans cet article, le soufflage est utilisé pour destructurer ou faire éclater les structures tourbillonnaires de lunette arrière dans la perspective d'accroître les pressions statiques pariétales et par conséquent réduire la traînée aérodynamique. Contrairement aux structures tourbillonnaires de type sillage qui prennent naissance au niveau des montants latéraux de pare-brise, les structures tourbillonnaires de lunette arrière sont de type jet.

L'éclatement tourbillonnaire s'obtient ici en augmentant la valeur du nombre de Swirl (rapport de la vitesse azimutale sur la vitesse relevée dans la direction de l'axe tourbillonnaire) à partir de l'accroissement des vitesses azimutales locales jusqu'à des valeurs proches de 1,5 [20]. Pour toutes les configurations, les nombres de Swirl sont déterminés à partir de mesures de vitesses relevées par vélocimétrie laser 3 composantes dans des plans $\mathrm{P}$ perpendiculaires à l'axe tourbillonnaire, figure 17 . Sur cet axe et de part et d'autre du point E (Fig. 17), les valeurs maximales de vorticité $\omega$ relevées sans soufflage décroissent respectivement de $1,00 \times 10^{4} \mathrm{~s}^{-1}$ à $0,25 \times 10^{4} \mathrm{~s}^{-1}$. Comme pour l'aspiration, le soufflage s'effectue dans une direction perpendiculaire au plan de la lunette arrière à partir d'une fente d'épaisseur $e=10^{-3} \mathrm{~m}$ positionnée sur toute sa hauteur, figure 16. L'analyse s'effectue pour les vitesses de soufflage $V_{\mathrm{s}}=0,5 V_{0}, V_{0}$ et $1,5 V_{0}$ où $V_{0}$ désigne la vitesse de l'écoulement incident. L'efficacité du contrôle est

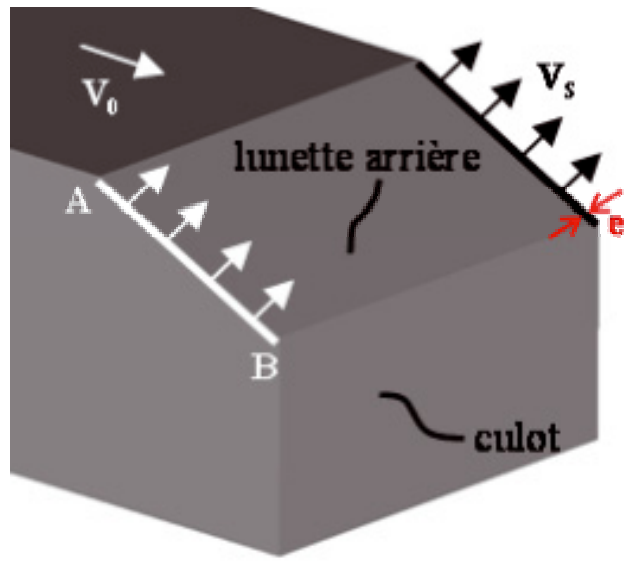

Fig. 16. Lunette arrière corps de Ahmed avec soufflages latéraux perpendiculaires à la paroi [18].

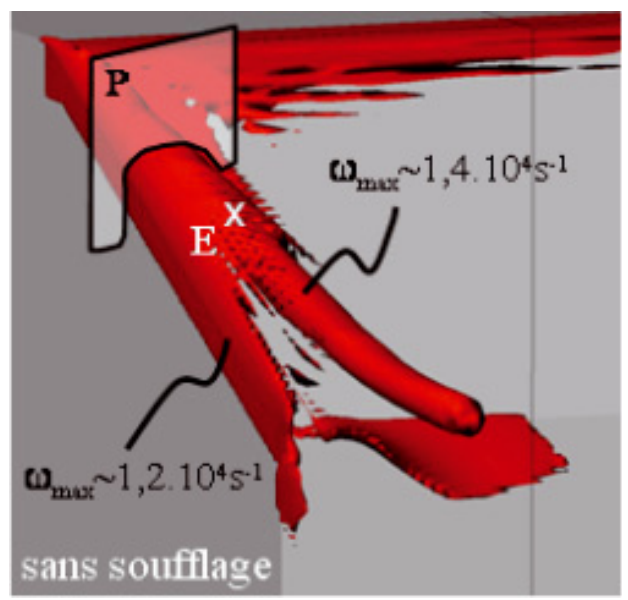

Fig. 17. Surface d'égale vorticité $\left(\omega=5000 \mathrm{~s}^{-1}\right)$ avec valeurs maximales relevées sans soufflage [18]. P, plan courant de mesure de la vorticité, E un point sur l'axe tourbillonnaire. Résultats numériques 3D lunette arrière corps de Ahmed.

analysée à partir des surfaces d'égale vorticité, des distributions de pression statique sur la lunette arrière et des réductions de traînée aérodynamique.

Pour toutes les vitesses de soufflage, la structure tourbillonnaire longitudinale et la distribution pariétale de pression statique sur la lunette arrière évoluent et des réductions significatives de traînée sont relevées pour les vitesses de soufflage $V_{\mathrm{s}}=0,5 V_{0}, V_{0}$ et $1,5 V_{0}$ [21], voir figures 17-21. La structure tourbillonnaire passe de l'état déstructuré à l'état éclaté avec recirculation et courant de retour avec point d'arrêt lorsque la vitesse de soufflage évolue de 0,5 à 1 et $1,5 V_{0}$ [21]. Pour toutes ces vitesses, les valeurs des pressions statiques pariétales relevées sous la structure tourbillonnaire augmentent et les maximums de pression statique sont relevés pour la vitesse de soufflage $V_{\mathrm{s}}=0,5 V_{0}$, figures 18 et 21 . D'un point de vue énergétique, la meilleure efficacité énergétique s'obtient pour une vitesse de soufflage proche de $0,5 V_{0}$. Des expérimentations réalisées en partenariat avec le 


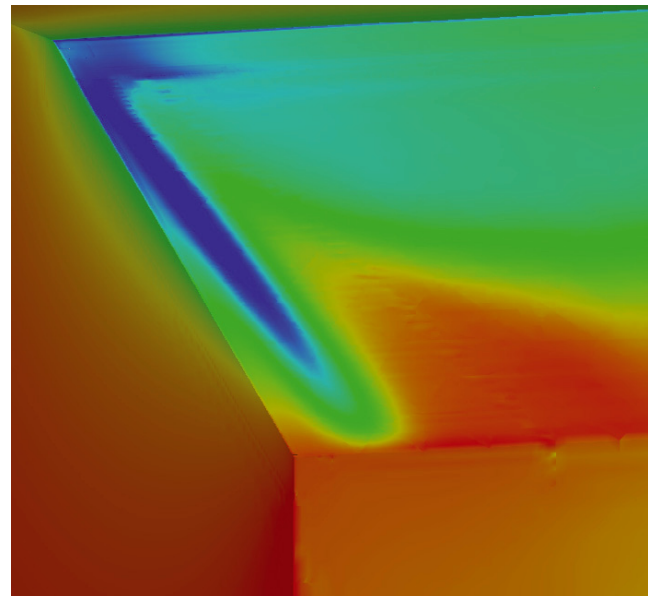

Fig. 18. Coefficients pariétaux de pression statique $C_{\mathrm{p}}$ (de bleu à rouge valeurs entre -1 et 0 ) sur lunette arrière relevés sans soufflage [18]. Résultats numériques 3D lunette arrière corps de Ahmed.

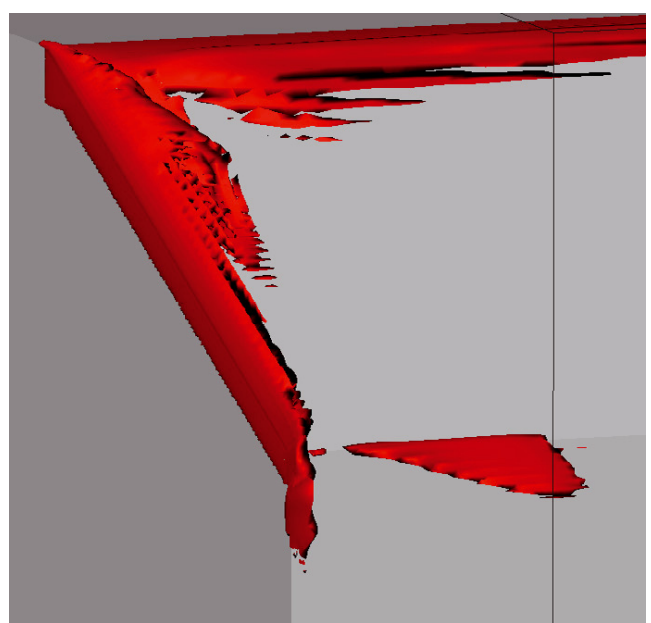

Fig. 19. Surface d'égale vorticité $\left(\omega=5000 \mathrm{~s}^{-1}\right)$ avec soufflage $V_{\mathrm{s}}=0,5 V_{0}, \Delta C_{x}=-5,6 \%$ [18]. Résultats numériques $3 \mathrm{D}$ lunette arrière corps de Ahmed.

Laboratoire de Mécanique et d'Énergétique d'Orléans sur une configuration analogue confirment l'éclatement tourbillonnaire et les remontées de pression sur la lunette arrière (article Mécanique \& Industrie à paraître).

En l'absence de solutions géométriques permettant de réduire ou de supprimer totalement les structures tourbillonnaires de lunette arrière sans incidence forte sur l'habitabilité des véhicules (garde au toit, déplacements latéraux), ces résultats montrent l'intérêt de l'éclatement tourbillonnaire pour remonter les pressions de culot et réduire la traînée des véhicules automobiles. Des travaux sont en cours pour accroître l'efficacité du contrôle et réduire la contribution énergétique nécessaire à l'apparition de l'éclatement. Lorsque les solutions auront été identifiées, le travail suivant consistera alors à mettre au point et développer des actionneurs de petites tailles et de faible masse (MEMS électriques, électromagnétiques ou autres) à haut rendement énergétique. Des collaborations

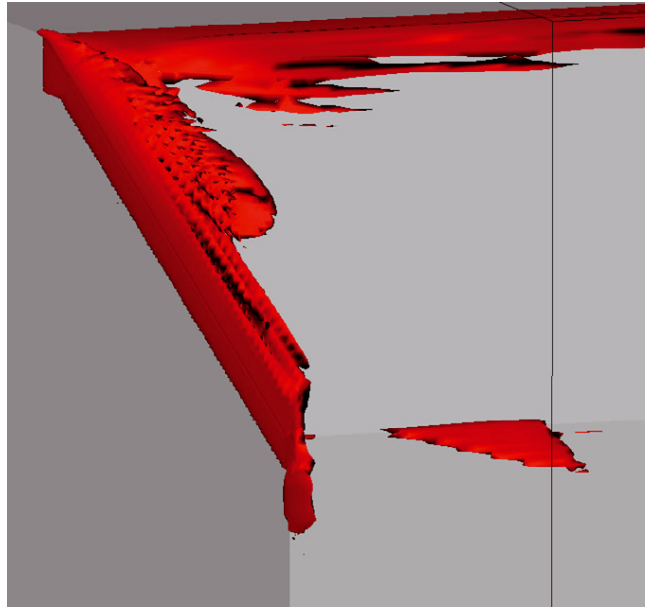

Fig. 20. Surface d'égale vorticité $\left(\omega=5000 \mathrm{~s}^{-1}\right)$ avec soufflage $V_{\mathrm{s}}=V_{0}, \Delta C_{x}=-6,2 \%$ [18]. Résultats numériques 3D lunette arrière corps de Ahmed.

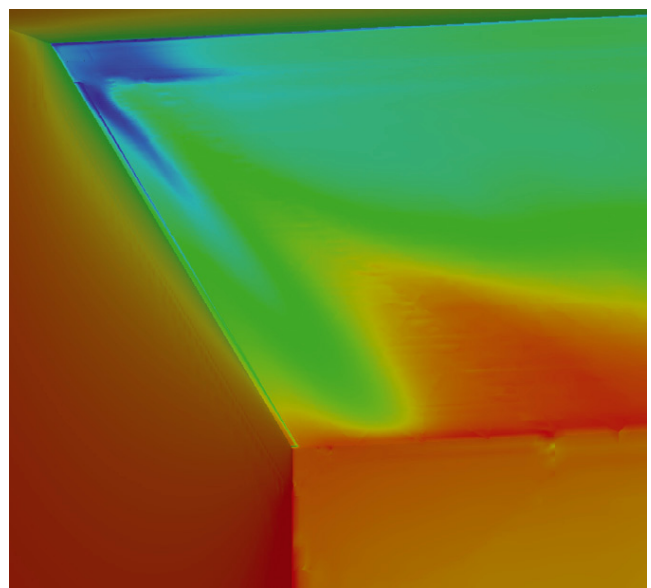

Fig. 21. Coefficients de pression statique $C_{\mathrm{p}}$ (de bleu à rouge valeurs entre -1 et 0 ) pour soufflage $V_{\mathrm{s}}=0,5 V_{0}$. À comparer avec la figure 18 [18]. Résultats numériques 3D lunette arrière corps de Ahmed.

sont déjà engagées avec l'IEMN, le LEMAC et la société FLOWDIT pour développer de tels systèmes.

\subsubsection{Solution par soufflage et aspiration alternés à débit moyen nul (jet synthétique)}

Le contrôle des écoulements et des décollements aérodynamiques par jets alternés de types soufflé et aspiré offre des perspectives intéressantes en termes d'efficacité énergétique. Le jet est dit pulsé ou synthétique selon que la moyenne temporelle est respectivement non nulle ou nulle sur une période [22]. Dans cet article, seuls les travaux et résultats récents obtenus dans le cadre d'un contrôle par jet synthétique sont présentés [22-24]. Ces travaux sont effectués à partir des expertises développées autour du contrôle par aspiration et soufflage continus décrits aux paragraphes précédents. La géométrie utilisée reste identique et les résultats relevés sur la configuration 


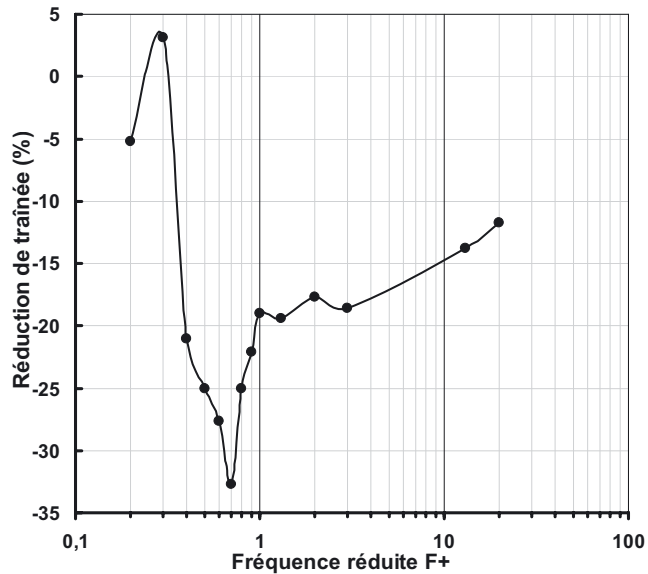

Fig. 22. Pourcentage de réduction du coefficient $C_{x}$ en fonction de la fréquence réduite $F^{+}$pour le coefficient de quantité de mouvement $C_{\mu}=2,4 \times 10^{-4}[26]$, résultats numériques $2 \mathrm{D}$ corps de Ahmed.

3D sont obtenus après avoir développé un savoir-faire sur des configurations bidimensionnelles.

La fréquence du jet synthétique s'obtient à partir des grandeurs caractéristiques de Kolmogorov définies à partir de la hauteur de la géométrie et de la vitesse de l'écoulement incident $V_{0}=40 \mathrm{~m} . \mathrm{s}^{-1}$. Pour ces valeurs, la fréquence caractéristique $F_{\mathrm{k}}$ est égale à $140 \mathrm{~Hz}$. Le coefficient de quantité de mouvement $C_{\mu}$ est déterminé à partir du maître couple de la géométrie, de la surface en plan de la fente du jet synthétique, de l'amplitude de la vitesse maximale du jet synthétique et de la vitesse de l'écoulement $V_{0}$. La fréquence du jet synthétique $F_{\mathrm{j}}$ et la fréquence caractéristique $F_{\mathrm{k}}$ permettent alors de définir une fréquence réduite notée $F^{+}$à partir du rapport $F_{\mathrm{j}} / F_{\mathrm{K}}$.

Les résultats numériques obtenus sur une configuration bidimensionnelle du corps de Ahmed font apparaître deux régimes associés à des réductions significatives de traînée aérodynamique [26]. Un premier régime stationnaire $\left(F^{+}=20\right.$, Fig. 22$)$ permet de réduire et de repousser en aval du culot les valeurs maximales de pertes d'énergie volumique et un second régime $\left(F^{+}=0,7\right.$, Fig. 22), désigné dynamique, pour lequel la topologie de sillage est caractérisée par la formation de petites structures tourbillonnaires dont la fréquence d'émission apparait synchronisée avec la fréquence du jet synthétique [27]. Pour ce dernier régime, la valeur du coefficient de quantité de mouvement évolue sans incidence rapide sur la structure de l'écoulement et la valeur du coefficient de traînée aérodynamique, figure 23. La meilleure efficacité s'obtient lorsque la quantité de mouvement est introduite au cœur de la sous-couche logarithmique [28]. Les réductions de traînée relevées pour ces deux régimes sont respectivement de 13 (pour $F^{+}=20$ ) et $33 \%$ (pour $F^{+}=0,7$ ) [27], figures 22 et 23 .

Pour une configuration tridimensionnelle, les résultats de calculs relevés sans contrôle mettent en évidence deux régimes caractéristiques d'agitation tourbillonnaire [27]. Le premier régime est associé à l'émission d'allées

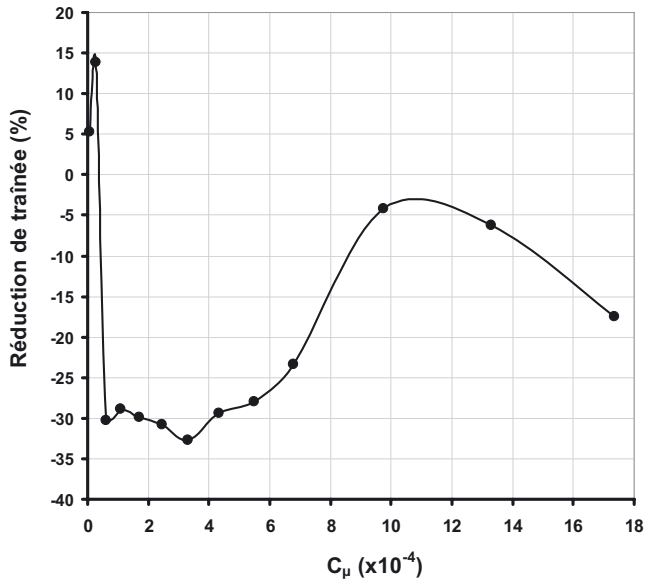

Fig. 23. Pourcentage de réduction du coefficient $C_{x}$ en fonction du coefficient de quantité de mouvement $C_{\mu}$ pour la fréquence réduite $F^{+}=0,7$ [26], résultats numériques $2 \mathrm{D}$ corps de Ahmed.

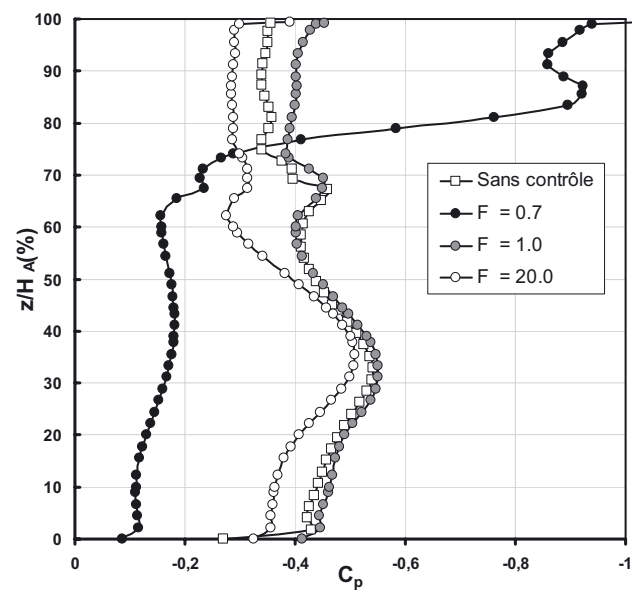

Fig. 24. Distribution des coefficients de pression statique $C_{\mathrm{p}}$ sur la lunette arrière et le culot sans et avec contrôle pour $C_{\mu}=2,4 \times 10^{-4}$ et les fréquences réduites $F^{+}=0,7,1,0$ et 20,0 [26], résultats numériques $2 \mathrm{D}$ corps de Ahmed.

tourbillonnaires de Von Karman à partir du soubassement et est peu influencé par l'écoulement de lunette arrière. Le second régime est tel que les fréquences des émissions tourbillonnaires issues de la lunette arrière et du soubassement apparaissent en phase. À ces modes sont respectivement associés les nombres de Strouhal 0,4 et 0,7 . Pour la fréquence réduite $F^{+}=0,7$ et un contrôle positionné en fin de pavillon en amont du décollement $\left(4,5 \times 10^{-3} \mathrm{~m}\right)$, la traînée aérodynamique diminue avec l'accroissement du coefficient de quantité de mouvement $C_{\mu}$ jusqu'à une valeur seuil $C_{\mu \mathrm{o}}$, voir figure 27 . Le développement transversal du décollement de lunette arrière issu de la fin du pavillon diminue (Figs. 25 et 26), l'écoulement en proche paroi s'organise à partir de distributions de rouleaux tourbillonnaires, les positions des centres tourbillonnaires de sillage se symétrisent dans le plan de symétrie longitudinal et la pression au culot augmente. Sur la lunette arrière et comme en 2D, 


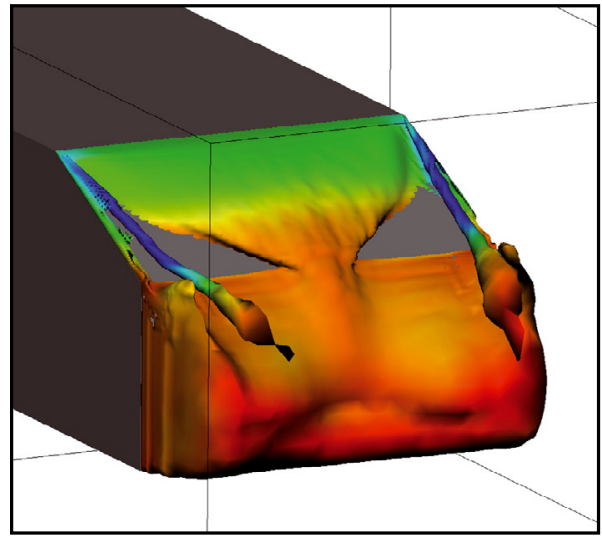

Fig. 25. Surfaces d'égales pertes d'énergie volumique $\left(C_{\mathrm{pi}}=1\right)$ coloriées par les valeurs des coefficients de pression statique $C_{\mathrm{p}}$, résultats numériques $3 \mathrm{D}$ corps de Ahmed relevés sans contrôle [27].

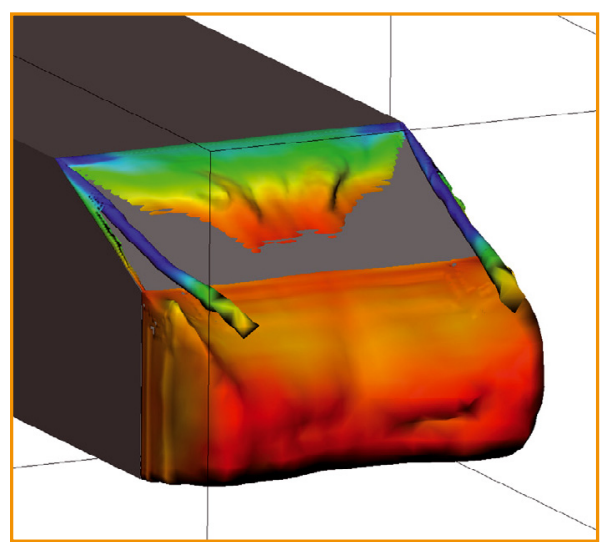

Fig. 26. Surfaces d'égales pertes d'énergie volumique $\left(C_{\mathrm{pi}}=1\right)$ coloriées par les valeurs des coefficients de pression statique $C_{\mathrm{p}}$, résultats numériques $3 \mathrm{D}$ corps de Ahmed relevés avec contrôle positionné en amont du décollement, $F^{+}=0,7$ et $C_{\mu}=4,2 \times 10^{-4}[27]$.

l'écoulement devient dynamique et l'apparition puis le transport des rouleaux tourbillonnaires s'effectuent en phase avec la fréquence du jet synthétique. Pour les configurations testées et le soufflage continu, les meilleures réductions de traînée s'obtiennent lorsque l'orifice transversal du contrôle est positionné en aval mais au plus près du décollement (intersection de la fin du pavillon avec le haut de la lunette arrière). La meilleure réduction de traînée proche de $13 \%$ s'obtient pour la même valeur $C_{\mu}=4,2 \times 10^{-4}$ du coefficient de quantité de mouvement, figure 27 [27].

Ces résultats obtenus par voie numérique sont comparés à des résultats expérimentaux réalisés en soufflerie sur une maquette de géométrie identique à échelle 0,7 [27]. Le jet synthétique s'obtient à partir d'une barrette d'actionneurs à membranes piézoélectriques montés en parallèle [29] et l'orifice du dispositif de contrôle est positionné à $-4,2$ ou $4 \times 10^{-3} \mathrm{~m}$ en amont ou en aval du décollement (fin du pavillon ou haut de la lunette arrière, Fig. 28). Pour la fréquence de résonnance des

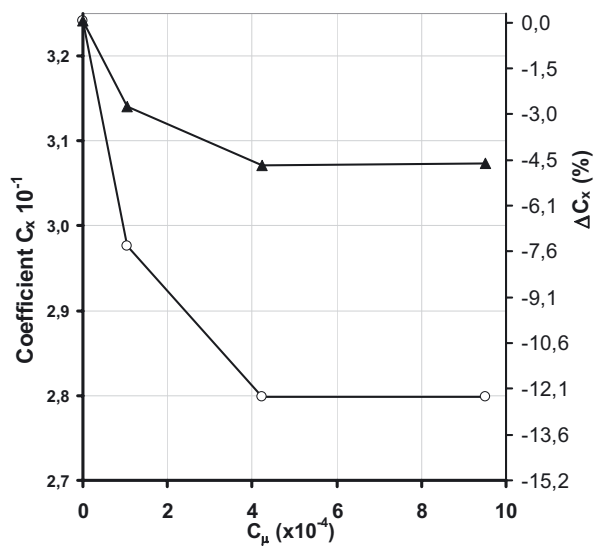

Fig. 27. Réduction du coefficient de traînée $C_{x}$ et pourcentage de réduction pour des fentes positionnées en amont et en aval du décollement. Résultats numériques 3D corps de Ahmed, d'après [27].

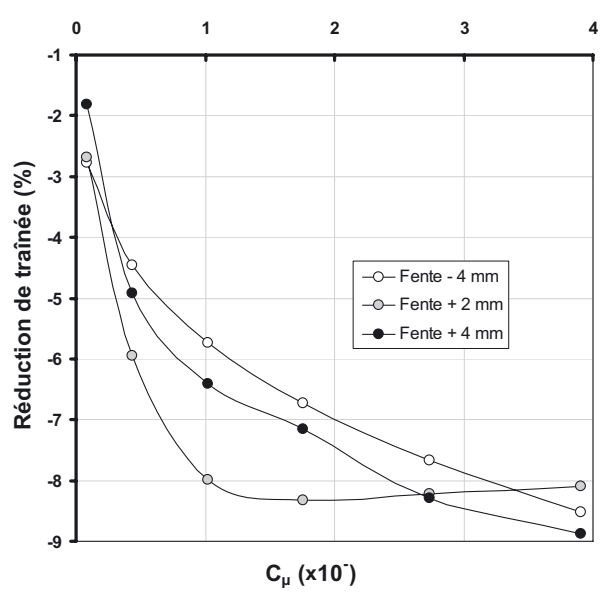

Fig. 28. Réduction du coefficient de traînée aérodynamique $C_{x}$ en fonction du coefficient de quantité de mouvement $C_{\mu}$ pour des fentes positionnées à $-4,+2$ et $+4 \mathrm{~mm}$. Résultats expérimentaux 3D corps de Ahmed, $R e=1,2 \times 10^{6}$ [27].

membranes des actionneurs $(F=520 \mathrm{~Hz})$ associée à la fréquence réduite $F^{+}=4,2$ et des largeurs de fente comprises entre 0,5 et $1,5 \times 10^{-3} \mathrm{~m}$, des vitesses maximales comprises entre 14 et $19 \mathrm{~m} . \mathrm{s}^{-1}$ sont relevées à la sortie des actionneurs. Comme précédemment (aspiration continue et jet synthétique 2D), les meilleures réductions de traînée s'obtiennent pour une fente positionnée en aval et au plus près de la ligne de décollement $\left(+2 \times 10^{-3} \mathrm{~m}\right.$, voir Fig. 28). Pour des valeurs croissantes du coefficient de quantité de mouvement comprises entre 0 et $3,9 \times 10^{-4}$, la traînée aérodynamique diminue rapidement pour croître légèrement et tendre vers $8 \%$ lorsque le coefficient de quantité de mouvement tend vers $3,9 \times 10^{-4}$. La réduction maximale représente $8,5 \%$ de la traînée aérodynamique relevée sans contrôle, figure 28 .

Au-delà du coefficient de quantité de mouvement $C_{\mu}=3,9 \times 10^{-4}$, la traînée aérodynamique n'évolue plus. Cette réduction résulte d'une diminution progressive de l'extension transversale de la région décollée sur le haut de lunette arrière qui s'accompagne d'un accroissement 
de la vitesse longitudinale au bas de la lunette arrière. Cette augmentation locale de la vitesse de transport reporte vers l'aval la partie supérieure de la structure tourbillonnaire torique de culot, les vitesses au voisinage du culot diminuent, les pressions statiques augmentent et la contribution du culot à la traînée aérodynamique décroît. De plus, l'écart des vitesses entre les parties inférieures de la lunette arrière et du culot se réduit et la structure tourbillonnaire de culot se symétrise. Enfin, le contrôle réduit l'activité spectrale associée au battement du bulbe décollée de lunette arrière, introduit une instabilité forcée qui contraint l'évolution instationnaire du sillage et renforce l'organisation autour de l'instabilité de Von Karman.

\section{Impact sur les consommations et les émissions}

Ces résultats montrent que des solutions en rupture existent pour réduire d'au moins $10 \%$ la traînée aérodynamique des véhicules automobiles. Ces solutions pourraient être utilisées dans des régions où subsistent des décollements ou lorsque les contraintes d'architecture et/ou de design ne permettent pas d'appliquer les préconisations aérodynamiques classiques. Elles offriraient ainsi des moyens supplémentaires aux équipes aérodynamiques chargées du développement des nouveaux véhicules. Pour des véhicules de gamme moyenne du type Mégane, la consommation diminuerait alors de $0,8 \mathrm{l}$ sur $100 \mathrm{~km}$ pour des trajets effectués à la vitesse stabilisée de $130 \mathrm{~km} \cdot \mathrm{h}^{-1}$. Les émissions de dioxyde de carbone seraient à cette vitesse réduites de $20 \mathrm{~g}$ par kilomètre et de $3,5 \mathrm{~g} . \mathrm{km}^{-1}$ sur le parcours NEDC.

Ces solutions nécessitent cependant des systèmes embarqués qui engendrent des accroissements de masse et de puissance électrique pour les systèmes actifs. Ces accroissements induisent des augmentations de consommation et d'émission de $\mathrm{CO}_{2}$ qui sont prises en compte dans les valeurs reportées dans le tableau 1.

Des associations de solutions peuvent enfin être imaginées (soufflage et jets synthétiques...) pour des réductions de $20 \%$ de la traînée aérodynamique sans incidence sur l'habitabilité et le confort des véhicules. De telles associations permettraient alors de diminuer les émissions de gaz carbonique de $7 \mathrm{~g} \cdot \mathrm{km}^{-1}$ sur le parcours NEDC et de 1,6 l la consommation sur $100 \mathrm{~km}$ à $130 \mathrm{~km} \cdot \mathrm{h}^{-1}$. Ces perspectives confirment l'intérêt du contrôle aérodynamique pour optimiser les rendements énergétiques des solutions actuelles et futures de transport (solutions hybride, hydrogène ou électrique).

\section{Conclusion et perspectives}

Les résultats présentés dans cet article montrent déjà des progrès significatifs et permettent d'envisager l'introduction du contrôle actif comme une des solutions pour réduire la consommation et les émissions de gaz à effet de
Tableau 1. Impacts estimés des solutions de contrôle sur la consommation à $130 \mathrm{~km} \cdot \mathrm{h}^{-1}$ et les émissions de $\mathrm{Co}_{2}$ pour le cycle NEDC. Valeurs déterminées pour $10 \%$ de réduction du coefficient de traînée aérodynamique $C_{x}$.

\begin{tabular}{|r|c|c|}
\hline Solutions & $\begin{array}{c}\text { Réduction } \\
\text { consommation }\end{array}$ & $\begin{array}{c}\text { Réduction } \\
\text { émission } \\
\mathrm{CO} 2 \\
\text { g.km }^{-1} \\
(\mathrm{NEDC})\end{array}$ \\
\hline Contrôle passif & $\begin{array}{c}1 / 100 \mathrm{~km} \text { à } \\
130 \mathrm{~km} \cdot \mathrm{h}^{-1}\end{array}$ & 0,8 \\
\hline Elts Sép Longitudinaux & 0,8 & 2,5 \\
\hline Elts Sép Transversaux & 0,4 & 1,6 \\
\hline Vortex Générateurs & & \\
\hline Contrôle actif & 0,7 & 2,9 \\
\hline Aspiration continue LuAR & 0,7 & 2,4 \\
\hline Aspiration continue montant baie & 0,7 & 2,1 \\
\hline Soufflage continu culot droit & 0,4 & 1,5 \\
\hline Soufflage continue LuAR (éclt) & 0,8 & 3,1 \\
\hline Jets synthétiques LuAR & & \\
\hline
\end{tabular}

serre des véhicules automobiles. Ces solutions pourraient également être utilisées pour améliorer les performances des futurs véhicules hybrides et électriques qui devraient progressivement se substituer aux véhicules à moteur thermique. Les objectifs sont évidemment ambitieux et nécessitent de poursuivre le travail de recherche engagé pour améliorer la connaissance des phénomènes physiques, optimiser les protocoles de contrôle et concevoir en parallèle des actionneurs robustes à faible coût capables d'intégrer les exigences automobiles. Les programmes de recherche doivent alors s'appuyer sur l'ensemble des compétences scientifiques et techniques développées dans les laboratoires de recherche industriels et universitaires qui travaillent et échangent sur des thématiques identiques et connexes. C'est ainsi qu'a été créé sous l'impulsion de Renault, de PSA et du CNAM, un Centre National de Recherche Technologique désigné CNRT «Aérodynamique et Aéroacoustique des Véhicules Terrestres » et a été développé un Groupement de Recherche (GdR) intitulé « Contrôle des Décollements », voir les sites www. cnrt2a. asso.fr et www.imft.fr/GDR2502

S'agissant du Centre National de Recherche Technologique désigné « Aérodynamique \& Aéroacoustique des Véhicules Terrestres », Renault et PSA sont membres fondateurs et animent le comité scientifique. Leurs contributions consistent à identifier et prioriser les axes de recherche, rechercher les meilleurs partenaires et assurer le suivi scientifique des travaux financés par ce CNRT. Ces dernières années, la recherche s'est orientée vers l'identification de produits non toxiques destinés à améliorer le rendu des visualisations pariétales (IMFT, IAT) dans les régions à faible vitesse, l'analyse des écoulements de césures (LIMSI, LME Valenciennes, LEA Poitiers, Signal Développement) et la recherche de solutions destinées à mettre au point des nappes souples de capteurs pariétaux de pression statique (LAUM \& ESIEE). Les interactions sillage/décollement (LEA de Poitiers), le contrôle de décollements par plasma froid (université 
d'Orléans et LEA de Poitiers) et le lancement d'une action d'évaluation des actionneurs sur une maquette d'essais de type corps de Ahmed évoluée destinée à tester des dispositifs de contrôle des décollements sont des sujets actuellement en cours d'analyse ou d'étude. Des informations sont disponibles sur le site du CNRT R2A, www. cnrt2a. asso.fr

Des résultats significatifs ont également été obtenus par les acteurs du Groupement de Recherche GdR 2502 «Contrôle Des Décollements », dont les activités s'articulent autour de laboratoires publics (LEA Poitiers, IMFT, ONERA, Institut PRISME/LME Orléans, ENSAM...) et privés (SNECMA, Dassault, PSA, Renault et PlasticOmnium). Les travaux de recherche s'effectuent à partir de géométries simplifiées de types corps de Ahmed, profil d'aile (OAT15) ou diffuseur plan. De nombreux résultats ont été présentés dans le cadre de journées d'études et/ou publiés dans diverses revues ou actes de congrès. Les résultats obtenus de 2001 à 2005 et publiés au début de l'année 2006 dans un recueil CEPADUES, ont permis de reconduire cette organisation au début de l'année 2006 pour une nouvelle période de 4 années. Renault, PSA, Dassault et l'ONERA sont membres actifs du conseil scientifique de ce GdR, voir site www .imft.fr/GDR2502

En parallèle sont développées des collaborations scientifiques pour croiser les expériences, partager les moyens expérimentaux ou numériques et développer de nouvelles idées. Un projet PREDIT (collaborations Renault, PSA, université de Bordeaux, ESPCI, CNAM et Plastic Omnium) a ainsi récemment été déposé et accepté sur le thème du contrôle par jets pulsés, projet CARAVAJE. Des recherches devront être poursuivies sur le jet synthétique pour mettre en concurrence ces deux approches, mieux évaluer leurs potentiels, leurs avantages, leurs inconvénients et identifier leur efficacité. La réponse industrielle à la nécessité du contrôle des écoulements et des décollements aérodynamiques pour réduire la consommation et les émissions de gaz à effet de serre des automobiles sera issue de l'une de ces deux solutions. Les progrès récents réalisés sur les dimensions, les masses et l'efficacité des actionneurs de type MEMS associés au développement de l'expertise sur le contrôle aérodynamique permettent d'envisager l'intégration de ces solutions d'ici 2015.

Remerciements. Les auteurs remercient particulièrement l'ANRT ainsi que Mathieu Rouméas, Benjamin Lehugeur et Cédric Leclerc, ingénieurs et docteurs, pour leurs contributions à cet article effectuées dans le cadre de travaux de thèses CIFRE entre 2003 et 2008. Ces contributions ont permis de réaliser des avancées significatives pour imaginer et développer des solutions destinées à réduire la consommation et les gaz à effet de serre des futurs véhicules automobiles.

\section{Références}

[1] Passenger Cars, University of Sheffield and University of Michigan, Produced by SASI Group (Sheffield) and Mark Newmann (Michigan), Map031, 2006
[2] World Energy Outlook 2007, Executive Summary, China and India Insights, International Energy Agency IEA, ISBN: 978-92-64-02730-5

[3] F. Chometon, P. Gilliéron, Modélisation des écoulements tridimensionnels décollés autour des véhicules automobiles, Congrès SIA, Société des Ingénieurs de l'Automobile, $N^{\circ}$ SIA 96-09-11, Courbevoie/Paris, 5 et 6 novembre 1996, p. 10

[4] P. Gilliéron, F. Chometon, Reduction of cooling air drag of road vehicle: an analytical approach, SAE paper $\mathrm{N}^{\circ}$ 01B-48, SAE Congress, Detroit, Michigan, USA, March 2001

[5] T. Ivanic, P. Gilliéron, Reduction of the aerodynamic drag to cooling systems: an analytical and experimental approach, SAE paper $\mathrm{N}^{\circ}$ 2005-01-1017, SAE Congress, Detroit, Michigan, USA, April 2005

[6] F. Chometon, P. Gilliéron, Assessment of engine cooling performance by measurement of cooling airflow drag in aerodynamic wind tunnels, SAE Congress, Detroit, Michigan, USA, February 23-26, 1998

[7] F. Chometon, P. Gilliéron, Dépouillement assisté par ordinateur des visualisations pariétales en aérodynamique, Compte-rendus Académie des Sciences Paris, tome 319, série II, 1994, pp. 1149-1156

[8] S.R. Ahmed, G. Ramm, G. Faltin, Some salient features of the time-averaged ground vehicle wake, SAE technical paper series, $N^{\circ} 840300$, Detroit, 1984

[9] P. Gillieron, F. Chometon, Modelling of stationary three-dimensional detached airflows around an Ahmed Reference Body, Third International Workshop on Vortex, ESAIM, Proceedings, 7, 1999, pp. 173-182, http://www . emath.fr/proc/Vol7/

[10] P. Gilliéron, A. Spohn, Flow separations generated by a simplified geometry of an automotive vehicle, Congrès IUTAM Symposium on Unsteady Separated Flows, April 8-12, Toulouse, France 2002

[11] E. Levallois, P. Gilliéron, Contrôle des écoulements en aérodynamique automobile \& réduction de traînée par éléments séparateurs - Analyse par PIV, Colloque National de Visualisation et de Traitement d'Images en Mécanique des Fluides, Fluvisu 11, Lyon, 6-9 juin 2005

[12] Renault, Direction de la Communication, Communiqué de Presse, À l'occasion du Challenge Bibendum Michelin, Renault présente Logan «Renault ECO2 » concept, véhicule écologique et économique, $1^{\mathrm{er}}$ octobre 2007

[13] C.H. Bruneau, P. Gilliéron, I. Mortazavi, Passive control around the square back Ahmed body using porous devices, J. Fluids Eng. (2007)

[14] M. Rouméas, P. Gilliéron, A. Kourta, Réduction de traînée par contrôle des décollements autour d'une géométrie simplifiée: Étude paramétrique $2 \mathrm{D}, 17^{\mathrm{e}}$ Congrès Français de Mécanique, 29 août au 2 septembre 2005, Troyes, France, 2005

[15] M. Rouméas, P. Gilliéron, A. Kourta, 2005, Analyzis and control of the near-wake flow over simplified car geometry, 4th Conference on Bluff Body Wakes and VortexInduced, Vibrations (BBVIV4), Santorin, Greece, 2124 June, 2005

[16] M. Rouméas, Contribution à l'analyse et au contrôle des sillages de corps épais par aspiration ou soufflage continu, thèse doctorat, CIFRE Renault/Institut National Polytechnique de Toulouse, juin 2006 
[17] B. Lehugeur, P. Gilliéron, Drag reduction by active control of A-pillar vortex breakdown on a simplified car geometry, European Drag Reduction and Flow Control Meeting, Ischia, Italy, 10-14th april 2006

[18] B. Lehugeur, Caractérisation et contrôle des structures tourbillonnaires longitudinales en aérodynamique automobile, thèse doctorat, CIFRE Renault/université Pierre et Marie Curie, mai 2007

[19] B. Lehugeur, P. Gilliéron, Analyse de l'influence du contrôle des tourbillons longitudinaux de montants de baie sur les champs de pression pariétale en aérodynamique automobile, $18^{\mathrm{e}}$ Congrès Français de Mécanique, AFM, Grenoble, France, du 27 au 31 août 2007

[20] P. Billant, J.M. Chomaz, P. Huerre, Experimental study of vortex breakdown in swirling jets, J. Fluid Mech. 376 (1998) 183-219

[21] B. Lehugeur, P. Gilliéron, T. Ivanic, Contribution de l'éclatement tourbillonnaire à la réduction de la traînée des véhicules automobiles : Approche numérique, Comptes Rendus de l'Académie des Sciences (CRAS), Section Mécanique, 334 (2006) 368-372

[22] B.L. Smith, A. Glezer, The formation and evolution of synthetic jets, Phys. Fluids 10 (1998) 2281-2297

[23] A. Glezer, M. Amitay, Synthetic jets, Annu. Rev. Fluid Mech. 34 (2002) 503-529
[24] Q. Gallas, On the modeling and design of zero-net mass flux actuators, Ph.D. thesis, University of Florida, Gainesville, 2005

[25] R. Raju, Q. Gallas, R. Mital, L. Cattafesta, Scaling of pressure drop of oscillatory flow through a slot, Phys. Fluids 19 (2007) 078107-078107-4

[26] C. Leclerc, E. Levallois, P. Gilliéron, A. Kourta, Aerodynamic Drag Reduction by Synthetic Jet: A 2D Numerical Study Around a Simplified Car, 3rd AIAA Flow Control Conference, San Francisco, California, 58 June 2006

[27] C. Leclerc, Réduction de la traînée d'un véhicule automobile simplifié à l'aide du contrôle actif par jet synthétique, thèse doctorat, CIFRE Renault/Institut de Mécanique des Fluides de Toulouse, janvier 2008

[28] C. Leclerc, E. Levallois, P. Gilliéron, A. Kourta, Q. Gallas, Phase locked analysis of a simplified car geometry wake flow control using synthetic jet, ASME Joint U.S., European Fluids Engineering Summer Meeting, paper N FEDSM2006-98469, July, Miami, Florida, 2006

[29] Q. Gallas, Modeling and Development of synthetic Jet Actuators in Flow Separation Control Application, IUTAM Symposium on Flow Control and MEMS, Londres, UK, 19-22 septembre 2006 